\title{
A Survey of the Relative Abundance of Mosquitoes in Their Preferred Breeding Micro-habitats in Refuse Dumps in Owerri, Imo State, Nigeria
}

\author{
Chidinma A. Ikpeama ${ }^{1, \star}$, Ifeanyi O. C. Obiajuru ${ }^{2}$, Bertram E. B. Nwoke ${ }^{1}$, Monica N. Ezike ${ }^{1}$ \\ ${ }^{1}$ Department of Animal and Environmental Biology, Imo State University, Nigeria \\ ${ }^{2}$ Department of Microbiology \& Parasitology, Faculty of Medicine, Imo State University, Nigeria
}

Copyright $\bigcirc 2017$ by authors, all rights reserved. Authors agree that this article remains permanently open access under the terms of the Creative Commons Attribution License 4.0 International License

\begin{abstract}
Studies on the abundance and preferred breeding sites of mosquitoes in different larval micro-habitats of refuse disposal dumps in Owerri were carried out between April and December 2016. Mosquito larvae were collected twice a week using standard dipping method and reared to adult stage. Two thousand, nine hundred and eighty seven adult mosquitoes were harvested. They were identified morpho-metrically into different species using binocular stereomicroscopes and taxonomic identification keys. Eight species of mosquitoes were identified: Aedes aegypti (28\%), Aedes albopictus (18.1\%), Anopheles species (4.0\%), Culex trigripes (16.0\%), Culex quinquefasciatus (19.9\%), Culex vittatus (5.3\%), Eretampodite spp (4.8\%) and Toxorhynchites spp (3.7\%). The findings showed that blocked gutters, empty cans and ground pools favoured the breeding of mosquitoes. Highest abundance of mosquitoes in the study sites were recorded in Relief Market and Concord Area. The presence of mosquitoes in the refuse dump sites was observed throughout the year. This suggests why mosquito borne diseases like malaria are prevalent in the study area throughout the year. The implication of the findings and recommendations for implementation of proper and regular disposal of refuse are discussed.
\end{abstract}

Keywords Relative Abundance, Mosquitoes, Breeding Microhabitats, Refuse Dumps Sites, Owerri

\section{Introduction}

Insect vectors are the largest group of disease transmitting agents [1]. In most cases, the widest diseases - spreading, mosquitoes are the commonest vectors of insect borne diseases [2]. Among a wide range of vector - borne disease, malaria and lymphatic filariasis stand out as serious health hazards in the contest of poor drainage.

The principal vector of filariasis (Culex quinquefasciatus) breeds in polluted water in ditches like tins and any kind of water accumulated in containers while anopheles mosquitoes are adapted to life in polluted water in urban environments [3]. In recent years the species have been found breeding in highly polluted water with high concentration of metal and human faces [4]. The mutual beneficial relationship between Culex quinquefasciatus, a non-malaria vector and Anopheles gambiae can lead to elevated malaria vector densities in urban environments [4]. Culex quinquefasciatus breeds efficiently in artificial sites like drainage facilities. Once they inhabit these sites, they create an environment in which Aedes gambiense were discovered in gutters blocked by refuse and sewage already inhabited by Culex species [4].

As a result of improved health facilities and increased awareness, urbanization is generally expected to reduce malaria transmission but on the contrary, the disease still persists in African cities. In some cases, the prevalence is higher than in rural areas, $[2,5]$. There is presently a rapid growth in population of Owerri due to rural/urban migration. This has given rise to a high generation of solid wastes from industries, homes, schools and markets. Many African cities like Owerri do not have efficient waste collection and disposal services [6]. In Nigeria, the sources of solid wastes are commercial centres, industries, households, agricultural and educational establishments. Effective disposal program for these solid wastes is lacking. This poor disposal habit is associated with spread of vector - borne diseases like malaria and dengue fever in the country [5]. Often times, solid wastes that are improperly disposed become breeding sites for mosquitoes due to pools of rain water collected in discarded solid wastes such as cans, bottles and used car tires.

The present study was undertaken to determine the relative abundance of various mosquito - vectors and their distribution in undisposed refuse dumps existing in different parts of Owerri. This will help to determine the impact of 
man-made ecosystem on the abundance and occurrence of disease vectors. The study will also help to highlight the effects of delays in disposal of refuse / wastes on the prevalence of disease - causing vectors as well as suggest possible surveillances approach to control the disease causing vectors.

\section{Materials and Methods}

\section{Study Area}

The study was conducted in Owerri metropolis, Imo State between April and December 2016. Owerri metropolis comprises of the urban areas of Owerri West L.G.A, Owerri North L.G.A and Owerri Municipal Council. This covers an area of 100 square kilometers and an estimated population of about 750,000 . It is located between latitude $5^{\circ} 29^{\prime} \mathrm{N}$ and longitude $7^{\circ} 2$ ' $\mathrm{E}$. The population comprised mostly of civil servants, traders, farmers, artisans, students and school leavers.

\section{Sample Technique}

Field investigations were carried out in 10 selected waste dump sites with differences in ages, sizes and locations namely: Relief Market area, Amkohia area, Orji area, Irette area, World Bank area, Egbu, Nekede area, Ikenegbu/ Aladinma area, Concord Hotel area and Douglas Road area. The refuse dump sites in the study area were composed of metals, tins, plastics, organic wastes (vegetable wastes, rotten food materials etc.) and industrial wastes. The refuse dumps were always damp throughout the study period.

\section{Collection and Rearing of Larvae}

Weekly surveys were carried out to determine the presence of stagnant water habitats, their characteristics and larval densities. Mosquito larvae were collected from their breeding microhabitats in the refuse dump sites between $7 \mathrm{am}$ and $11 \mathrm{am}$, twice a month. They were collected using a standard mosquito dipper (Clark USA) as described by [6]. Number of dips taken from each habitat was dependent on the perimeter of the larval habitat. The larval habitats were categorized depending on their perimeter and grouped into 3 classes; $\leq 10 \mathrm{~m}, 10-100 \mathrm{~m}$ and $>100 \mathrm{~m}$. A maximum of 10,50 and 100 dips were taken from these habitats respectively. Larvae were preserved in plastic jugs and taken to the laboratory for rearing according to [7]. They were kept at room temperature and fed with ground fish powder diet in aquarium. Adults that emerged were harvested by anesthetizing using pyrethrum base aerosol - Killit ${ }^{\circledR}$.

\section{Sample Identification}

The mosquitoes collected were examined one after the other using a binocular stereomicroscope with $\mathrm{x} 40$ objective and $\mathrm{x} 10$ eye piece. They were identified using their characteristic features such as size and length of antenna, size of maxillary palps, color of the maxillary palps, length of proboscis, shape of abdomen, color of abdomen, color of wings and legs etc., as in the keys of $[8,9,10,11 \& 12]$.

\section{Data Analysis}

Data obtained in this study were analyzed using simple percentage, chi statistics and presented in tables.

\section{Results}

A total of 2,987 mosquitoes were collected from the study sites. The highest number of collections $432(14.5 \%)$ was made in the month of April, followed by May (12.6\%) while the least abundance of mosquitoes was in November. 244 (8.2\%). Site 1 (Relief Market) recorded the highest number of mosquitoes 401 (13.42\%) followed by site 2 (Amakohia) with a total number of $332(11.1 \%)$ mosquitoes. The least collection $196(6.5 \%)$ was recorded in site 9. Table 1 summarizes the abundance of mosquito species in different refuse dump sites in Owerri metropolis. Table 2, shows mosquitoes' preference to the different microhabitats in the study sites Aedes albopictus and Aedes aegypti occurred mostly in containers and tires. Anopheles species and Culex species were mostly collected in ground pools and blocked drainage (gutters), mostly plant parts such as leaf petioles and tree holes recorded a high occurrence of Aedes species. Of all the microhabitats, blocked drainages (gutters) recorded the highest collection of mosquitoes.

Monthly distribution/occurrence of mosquitoes that breed in ground, pools (table 3) was highest during the month of April (75), followed by October (71) and least in November. Plastic containers had the highest occurrence during the month of May. In blocked gutter microhabitats, mosquitoes were highest in abundance $121(34.1 \%)$ during the month of September and June $100(23.5 \%)$ and closely followed by October 99 (27.9\%).

Table 4 summarizes the monthly - related prevalence of mosquitoes species identified. As shown, a total of 8 mosquito species were identified, they include Anopheles gambiae complex, Culex tigripis, Culex quinquefasciatus, Aedes albopictus, Culex vittatus Aedes aegypti, Eretampodites species, Toxorhynchites species. Aedes aegypti has the highest collection of $840(21.8 \%)$. This was followed by Culex quinquefasciatus 595 (19.5\%) while the least in abundance was Toxorhynchites species with a total number of $110(3.68 \%)$ individuals were recorded. However Anopheles gambiae complex was generally low in abundance in the study area during the period of study (120), Aedes species was most abundant at the peak of the rains, unlike the other species. 
Table 1. Abundance of Different species of mosquitoes in refuse dump sites in Owerri

\begin{tabular}{|c|c|c|c|c|c|c|c|c|c|c|}
\hline \multirow{2}{*}{$\begin{array}{c}\text { Refuse } \\
\text { Dump site }\end{array}$} & \multicolumn{10}{|c|}{ Occurrence of Mosquitoes } \\
\hline & April & May & June & July & August & September & October & November & December & Total \\
\hline $\begin{array}{c}\text { Relief } \\
\text { Market and } \\
\text { Environs } \\
\end{array}$ & $\begin{array}{c}58 \\
14.4 \%\end{array}$ & $\begin{array}{c}49 \\
12.22 \%\end{array}$ & $\begin{array}{c}46 \\
11.47 \%\end{array}$ & $\begin{array}{c}38 \\
9.48 \%\end{array}$ & $\begin{array}{c}32 \\
7.98 \%\end{array}$ & $\begin{array}{c}51 \\
12.7 \%\end{array}$ & $\begin{array}{c}52 \\
12.97 \%\end{array}$ & $\begin{array}{c}40 \\
9.98 \%\end{array}$ & $\begin{array}{c}35 \\
8.73 \%\end{array}$ & $\begin{array}{c}401 \\
99.9 \%\end{array}$ \\
\hline Amakohia & $\begin{array}{c}43 \\
12.95 \%\end{array}$ & $\begin{array}{c}47 \\
14.16 \%\end{array}$ & $\begin{array}{c}41 \\
12.35 \%\end{array}$ & $\begin{array}{c}31 \\
9.34 \%\end{array}$ & $\begin{array}{c}30 \\
9.04 \% \\
\end{array}$ & $\begin{array}{c}30 \\
9.04 \% \\
\end{array}$ & $\begin{array}{c}43 \\
12.95 \%\end{array}$ & $\begin{array}{c}32 \\
9.63 \% \\
\end{array}$ & $\begin{array}{c}35 \\
10.54 \%\end{array}$ & $\begin{array}{c}332 \\
100 \% \\
\end{array}$ \\
\hline Irete Area & $\begin{array}{c}46 \\
15.18 \%\end{array}$ & $\begin{array}{c}25 \\
8.25 \%\end{array}$ & $\begin{array}{c}26 \\
8.58 \%\end{array}$ & $\begin{array}{c}23 \\
7.59 \%\end{array}$ & $\begin{array}{c}37 \\
12.21 \%\end{array}$ & $\begin{array}{c}48 \\
15.84 \%\end{array}$ & $\begin{array}{c}39 \\
12.87 \%\end{array}$ & $\begin{array}{c}28 \\
9.24 \%\end{array}$ & $\begin{array}{c}31 \\
10.23 \%\end{array}$ & $\begin{array}{c}303 \\
99.9 \%\end{array}$ \\
\hline $\begin{array}{c}\text { World Bank } \\
\text { and } \\
\text { Environs }\end{array}$ & $\begin{array}{c}41 \\
13.75 \%\end{array}$ & $\begin{array}{c}36 \\
12.08 \%\end{array}$ & $\begin{array}{c}40 \\
13.42 \%\end{array}$ & $\begin{array}{c}36 \\
12.08 \\
\%\end{array}$ & $\begin{array}{c}32 \\
10.73 \%\end{array}$ & $\begin{array}{c}36 \\
12.08 \%\end{array}$ & $\begin{array}{c}32 \\
10.75 \%\end{array}$ & $\begin{array}{c}22 \\
7.38 \%\end{array}$ & $\begin{array}{c}23 \\
7.7 \%\end{array}$ & $\begin{array}{c}298 \\
99.97 \%\end{array}$ \\
\hline $\begin{array}{c}\text { Egbu Road } \\
\text { and } \\
\text { Environs }\end{array}$ & $\begin{array}{c}38 \\
12.75 \%\end{array}$ & $\begin{array}{c}39 \\
13.08 \%\end{array}$ & $\begin{array}{c}40 \\
13.42 \%\end{array}$ & $\begin{array}{c}30 \\
10.07 \\
\% \\
\end{array}$ & $\begin{array}{c}23 \\
7.71 \%\end{array}$ & $\begin{array}{c}40 \\
13.42 \%\end{array}$ & $\begin{array}{c}40 \\
13.42 \%\end{array}$ & $\begin{array}{c}30 \\
10.7 \%\end{array}$ & $\begin{array}{c}18 \\
6.04 \%\end{array}$ & $\begin{array}{c}298 \\
99.98 \%\end{array}$ \\
\hline Nekede Area & $\begin{array}{c}46 \\
16.02 \%\end{array}$ & $\begin{array}{c}37 \\
12.89 \%\end{array}$ & $\begin{array}{c}29 \\
10.1 \%\end{array}$ & $\begin{array}{c}31 \\
10.8 \%\end{array}$ & $\begin{array}{c}40 \\
13.937 \\
\%\end{array}$ & $\begin{array}{c}31 \\
10.8 \%\end{array}$ & $\begin{array}{c}42 \\
16.4 \%\end{array}$ & $\begin{array}{c}3 \\
1.05 \%\end{array}$ & $\begin{array}{c}28 \\
9.76 \%\end{array}$ & $\begin{array}{c}287 \\
99.98 \%\end{array}$ \\
\hline $\begin{array}{c}\text { IkenegbuAla } \\
\text { dinma }\end{array}$ & $\begin{array}{c}45 \\
16.34 \%\end{array}$ & $\begin{array}{c}35 \\
12.73 \%\end{array}$ & $\begin{array}{c}39 \\
14.18 \%\end{array}$ & $\begin{array}{c}20 \\
7.27 \%\end{array}$ & $\begin{array}{c}33 \\
12.00 \%\end{array}$ & $\begin{array}{c}35 \\
12.72 \%\end{array}$ & $\begin{array}{c}26 \\
9.45 \%\end{array}$ & $\begin{array}{c}21 \\
7.64 \%\end{array}$ & $\begin{array}{c}21 \\
7.64 \%\end{array}$ & $\begin{array}{c}275 \\
99.77 \%\end{array}$ \\
\hline $\begin{array}{c}\text { Concord and } \\
\text { Environs } \\
\end{array}$ & $\begin{array}{c}28 \\
14.29 \% \\
\end{array}$ & $\begin{array}{c}35 \\
17.86 \% \\
\end{array}$ & $\begin{array}{c}21 \\
10.71 \%\end{array}$ & $\begin{array}{c}12 \\
6.12 \% \\
\end{array}$ & $\begin{array}{c}28 \\
14.29 \% \\
\end{array}$ & $\begin{array}{c}23 \\
11.73 \% \\
\end{array}$ & $\begin{array}{c}20 \\
10.20 \% \\
\end{array}$ & $\begin{array}{c}10 \\
5.10 \% \\
\end{array}$ & $\begin{array}{c}19 \\
9.69 \% \\
\end{array}$ & $\begin{array}{c}196 \\
99.99 \% \\
\end{array}$ \\
\hline $\begin{array}{c}\text { Douglas } \\
\text { Area }\end{array}$ & $\begin{array}{c}47 \\
14.92 \%\end{array}$ & $\begin{array}{c}38 \\
12.06 \%\end{array}$ & $\begin{array}{c}50 \\
15.87 \%\end{array}$ & $\begin{array}{c}39 \\
12.38 \\
\% \\
\end{array}$ & $\begin{array}{c}35 \\
11.11 \%\end{array}$ & $\begin{array}{c}28 \\
8.89 \%\end{array}$ & $\begin{array}{c}28 \\
8.89 \%\end{array}$ & $\begin{array}{c}29 \\
9.21 \%\end{array}$ & $\begin{array}{c}21 \\
6.67 \%\end{array}$ & $\begin{array}{c}315 \\
100 \%\end{array}$ \\
\hline TOTAL & $\begin{array}{c}432 \\
14.46 \%\end{array}$ & $\begin{array}{c}377 \\
12.62 \%\end{array}$ & $\begin{array}{c}363 \\
12.15 \%\end{array}$ & $\begin{array}{c}293 \\
9.81 \%\end{array}$ & $\begin{array}{c}315 \\
10.55 \%\end{array}$ & $\begin{array}{c}355 \\
11.88 \%\end{array}$ & $\begin{array}{c}244 \\
11.88 \%\end{array}$ & $\begin{array}{c}354 \\
8.16 \%\end{array}$ & $\begin{array}{c}253 \\
8.47 \%\end{array}$ & $\begin{array}{c}2987 \\
99.98 \%\end{array}$ \\
\hline
\end{tabular}

Table 2. Preference of Micro-habitats by Mosquitoes species in Refuse dump sites

\begin{tabular}{|c|c|c|c|c|c|c|c|}
\hline \multirow[b]{2}{*}{ Mosquito species } & \multicolumn{6}{|c|}{ Micro-habitats in Refuse dump sites } & \multirow[b]{2}{*}{ Total } \\
\hline & Ground Poll & $\begin{array}{c}\text { Plastic } \\
\text { containers }\end{array}$ & $\begin{array}{c}\text { Metal } \\
\text { containers }\end{array}$ & Tires & $\begin{array}{c}\text { Living } \\
\text { Parts }\end{array}$ & $\begin{array}{l}\text { Blocked } \\
\text { Gutters }\end{array}$ & \\
\hline Aedes albopictus & $\begin{array}{c}16 \\
2.95 \%\end{array}$ & $\begin{array}{c}102 \\
18.81 \% \\
\end{array}$ & $\begin{array}{c}124 \\
22.87 \%\end{array}$ & $\begin{array}{c}204 \\
37.63 \% \\
\end{array}$ & $\begin{array}{c}66 \\
12.17 \% \\
\end{array}$ & $\begin{array}{c}30 \\
5.53 \%\end{array}$ & $\begin{array}{c}542 \\
99.97 \%\end{array}$ \\
\hline Aedes aegypti & $\begin{array}{c}23 \\
2.73 \% \\
\end{array}$ & $\begin{array}{c}194 \\
23.09 \% \\
\end{array}$ & $\begin{array}{c}193 \\
22.97 \% \\
\end{array}$ & $\begin{array}{c}263 \\
31.30 \% \\
\end{array}$ & $\begin{array}{c}101 \\
101 \% \\
\end{array}$ & $\begin{array}{c}66 \\
7.85 \% \\
\end{array}$ & $\begin{array}{c}840 \\
99.96 \% \\
\end{array}$ \\
\hline Culex trigripes & $\begin{array}{c}208 \\
43.60 \%\end{array}$ & $\begin{array}{c}43 \\
9.01 \%\end{array}$ & $\begin{array}{c}83 \\
17.40 \%\end{array}$ & $\begin{array}{c}47 \\
9.85 \%\end{array}$ & $\begin{array}{c}1 \\
0.20 \%\end{array}$ & $\begin{array}{c}95 \\
19.91 \%\end{array}$ & 477 \\
\hline Culex quinquefasciatus & $\begin{array}{c}146 \\
24.53 \% \\
\end{array}$ & $\begin{array}{c}62 \\
10.42 \% \\
\end{array}$ & $\begin{array}{c}65 \\
10.92 \% \\
\end{array}$ & $\begin{array}{c}16 \\
2.68 \% \\
\end{array}$ & - & $\begin{array}{c}306 \\
51.42 \% \\
\end{array}$ & $\begin{array}{c}595 \\
99.97 \% \\
\end{array}$ \\
\hline Culex vittatus & $\begin{array}{c}15 \\
9.43 \%\end{array}$ & $\begin{array}{c}59 \\
37.10 \% \\
\end{array}$ & $\begin{array}{c}27 \\
16.98 \% \\
\end{array}$ & $\begin{array}{c}2 \\
1.25 \% \\
\end{array}$ & $\begin{array}{c}11 \\
6.91 \% \\
\end{array}$ & $\begin{array}{c}45 \\
28.30 \% \\
\end{array}$ & $\begin{array}{c}159 \\
99.97 \%\end{array}$ \\
\hline Toxorhynchites species & - & - & - & $\begin{array}{c}35 \\
31.81 \% \\
\end{array}$ & $\begin{array}{c}13 \\
11.81 \% \\
\end{array}$ & $\begin{array}{c}62 \\
56.36 \% \\
\end{array}$ & $\begin{array}{c}110 \\
99.98 \% \\
\end{array}$ \\
\hline TOTAL & $\begin{array}{c}495 \\
16.57 \%\end{array}$ & $\begin{array}{c}492 \\
16.47 \%\end{array}$ & $\begin{array}{c}495 \\
16.57 \% \\
\end{array}$ & $\begin{array}{c}571 \\
19.11 \% \\
\end{array}$ & $\begin{array}{c}192 \\
6.42 \%\end{array}$ & $\begin{array}{c}742 \\
24.84 \% \\
\end{array}$ & $\begin{array}{c}2987 \\
99.98 \% \\
\end{array}$ \\
\hline
\end{tabular}


Table 3. Occurrence of Mosquitoes in the Different Micro-habitats

\begin{tabular}{|c|c|c|c|c|c|c|c|c|c|c|}
\hline \multirow{2}{*}{$\begin{array}{l}\text { Micro - } \\
\text { habitats }\end{array}$} & \multicolumn{9}{|c|}{ Monthly Occurrence of Mosquitoes } & \multirow{2}{*}{ Total } \\
\hline & April & May & June & July & August & September & October & November & December & \\
\hline $\begin{array}{l}\text { Ground } \\
\text { Pools }\end{array}$ & $\begin{array}{c}75 \\
15.15 \% \\
\end{array}$ & $\begin{array}{c}68 \\
13.73 \% \\
\end{array}$ & $\begin{array}{c}59 \\
11.91 \% \\
\end{array}$ & $\begin{array}{c}50 \\
10.10 \% \\
\end{array}$ & $\begin{array}{c}55 \\
11.11 \% \\
\end{array}$ & $\begin{array}{c}43 \\
8.68 \% \\
\end{array}$ & $\begin{array}{c}71 \\
14.34 \% \\
\end{array}$ & $\begin{array}{c}35 \\
7.07 \% \\
\end{array}$ & $\begin{array}{c}39 \\
7.68 \% \\
\end{array}$ & $\begin{array}{c}495 \\
99.77 \% \\
\end{array}$ \\
\hline $\begin{array}{c}\text { Plastic } \\
\text { Containers } \\
\text { (Breakables) }\end{array}$ & $\begin{array}{c}70 \\
14.22\end{array}$ & $\begin{array}{c}78 \\
15.85\end{array}$ & $\begin{array}{c}52 \\
10.56\end{array}$ & $\begin{array}{c}50 \\
10.16\end{array}$ & $\begin{array}{c}51 \\
10.36\end{array}$ & $\begin{array}{c}58 \\
11.78 \%\end{array}$ & $\begin{array}{c}55 \\
11.17 \%\end{array}$ & $\begin{array}{c}40 \\
8.13 \%\end{array}$ & $\begin{array}{c}38 \\
7.72 \%\end{array}$ & $\begin{array}{c}492 \\
99.95 \%\end{array}$ \\
\hline $\begin{array}{c}\text { Metal } \\
\text { Containers } \\
\end{array}$ & $\begin{array}{c}70 \\
14.14 \% \\
\end{array}$ & $\begin{array}{c}73 \\
14.74 \% \\
\end{array}$ & $\begin{array}{c}54 \\
10.90 \% \\
\end{array}$ & $\begin{array}{c}54 \\
10.90 \% \\
\end{array}$ & $\begin{array}{c}50 \\
10.10 \\
\end{array}$ & $\begin{array}{c}44 \\
8.88 \% \\
\end{array}$ & $\begin{array}{c}66 \\
13.33 \% \\
\end{array}$ & $\begin{array}{c}40 \\
8.08 \% \\
\end{array}$ & $\begin{array}{c}44 \\
8.88 \% \\
\end{array}$ & $\begin{array}{c}495 \\
99.95 \% \\
\end{array}$ \\
\hline Tires & $\begin{array}{c}83 \\
14.53 \% \\
\end{array}$ & $\begin{array}{c}60 \\
10.50 \% \\
\end{array}$ & $\begin{array}{c}74 \\
12.95 \% \\
\end{array}$ & $\begin{array}{c}48 \\
8.40 \% \\
\end{array}$ & $\begin{array}{c}80 \\
14.01 \% \\
\end{array}$ & $\begin{array}{c}66 \\
11.55 \% \\
\end{array}$ & $\begin{array}{c}34 \\
5.95 \% \\
\end{array}$ & $\begin{array}{c}59 \\
10.33 \% \\
\end{array}$ & $\begin{array}{c}67 \\
11.73 \% \\
\end{array}$ & $\begin{array}{c}571 \\
99.5 \% \\
\end{array}$ \\
\hline $\begin{array}{c}\text { Living parts } \\
\text { of plants }\end{array}$ & $\begin{array}{c}41 \\
21.35 \% \\
\end{array}$ & $\begin{array}{c}17 \\
8.85 \% \\
\end{array}$ & $\begin{array}{c}24 \\
12.5 \% \\
\end{array}$ & $\begin{array}{c}20 \\
10.41 \% \\
\end{array}$ & $\begin{array}{c}10 \\
5.20 \%\end{array}$ & $\begin{array}{c}23 \\
11.97 \% \\
\end{array}$ & $\begin{array}{c}30 \\
15.62 \% \\
\end{array}$ & $\begin{array}{c}12 \\
6.25 \% \\
\end{array}$ & $\begin{array}{c}15 \\
7.81 \%\end{array}$ & $\begin{array}{c}192 \\
99.96 \%\end{array}$ \\
\hline $\begin{array}{c}\text { Blocked } \\
\text { Gutters }\end{array}$ & $\begin{array}{c}93 \\
12.53 \% \\
\end{array}$ & $\begin{array}{c}81 \\
10.91 \% \\
\end{array}$ & $\begin{array}{c}100 \\
13.47 \% \\
\end{array}$ & $\begin{array}{c}71 \\
9.56 \% \\
\end{array}$ & $\begin{array}{c}69 \\
9.29 \% \\
\end{array}$ & $\begin{array}{c}121 \\
16.30 \% \\
\end{array}$ & $\begin{array}{c}99 \\
13.34 \% \\
\end{array}$ & $\begin{array}{c}58 \\
7.81 \% \\
\end{array}$ & $\begin{array}{c}50 \\
6.73 \% \\
\end{array}$ & $\begin{array}{c}742 \\
99.94 \% \\
\end{array}$ \\
\hline TOTAL & $\begin{array}{c}432 \\
14.46 \% \\
\end{array}$ & $\begin{array}{c}377 \\
12.62 \% \\
\end{array}$ & $\begin{array}{c}363 \\
12.15 \%\end{array}$ & $\begin{array}{c}293 \\
9.80 \% \\
\end{array}$ & $\begin{array}{c}315 \\
10.54 \% \\
\end{array}$ & $\begin{array}{c}355 \\
11.88 \% \\
\end{array}$ & $\begin{array}{c}355 \\
11.88 \% \\
\end{array}$ & $\begin{array}{c}244 \\
8.16 \% \\
\end{array}$ & $\begin{array}{c}253 \\
8.47 \% \\
\end{array}$ & $\begin{array}{c}2987 \\
99.96 \% \\
\end{array}$ \\
\hline
\end{tabular}

Table 4. Monthly related Prevalence of the Relative Abundance of Mosquito species in Refuse dump sites

\begin{tabular}{|c|c|c|c|c|c|c|c|c|c|c|}
\hline \multirow{2}{*}{$\begin{array}{l}\text { Mosquito } \\
\text { species }\end{array}$} & \multicolumn{9}{|c|}{ Relative Abundance of Mosquito species } & \multirow{2}{*}{ Total } \\
\hline & April & May & June & July & Aug & Sept. & Oct. & Nov. & Dec. & \\
\hline Aedes albopictus & $\begin{array}{c}88 \\
16.23 \%\end{array}$ & $\begin{array}{c}76 \\
14.02 \%\end{array}$ & $\begin{array}{c}60 \\
11.07 \%\end{array}$ & $\begin{array}{c}36 \\
6.64 \%\end{array}$ & $\begin{array}{c}61 \\
11.25 \%\end{array}$ & $\begin{array}{c}56 \\
10.33 \%\end{array}$ & $\begin{array}{c}61 \\
11.25 \%\end{array}$ & $\begin{array}{c}53 \\
9.77 \%\end{array}$ & $\begin{array}{c}51 \\
9.40 \%\end{array}$ & $\begin{array}{c}542 \\
99.96 \%\end{array}$ \\
\hline Aedes aegypti & $\begin{array}{c}74 \\
8.80 \% \\
\end{array}$ & $\begin{array}{c}88 \\
10.47 \% \\
\end{array}$ & $\begin{array}{c}115 \\
13.69 \% \\
\end{array}$ & $\begin{array}{c}119 \\
14.16 \% \\
\end{array}$ & $\begin{array}{c}85 \\
10.11 \% \\
\end{array}$ & $\begin{array}{c}135 \\
16.07 \% \\
\end{array}$ & $\begin{array}{c}109 \\
12.97 \% \\
\end{array}$ & $\begin{array}{c}57 \\
6.78 \% \\
\end{array}$ & $\begin{array}{c}58 \\
6.90 \% \\
\end{array}$ & $\begin{array}{c}840 \\
99.95 \% \\
\end{array}$ \\
\hline $\begin{array}{c}\text { Anopheles } \\
\text { species }\end{array}$ & $\begin{array}{c}34 \\
28.33 \%\end{array}$ & $\begin{array}{c}17 \\
14.16 \%\end{array}$ & $\begin{array}{c}26 \\
21.66 \%\end{array}$ & $\begin{array}{c}11 \\
9.16 \%\end{array}$ & $\begin{array}{c}12 \\
10 \%\end{array}$ & $\begin{array}{c}13 \\
10.83 \%\end{array}$ & - & $\begin{array}{c}2 \\
1.66 \%\end{array}$ & $\begin{array}{c}5 \\
4.16 \%\end{array}$ & $\begin{array}{c}120 \\
99.96 \%\end{array}$ \\
\hline Culex trigripes & $\begin{array}{c}46 \\
9.64 \% \\
\end{array}$ & $\begin{array}{c}64 \\
13.41 \% \\
\end{array}$ & $\begin{array}{c}70 \\
14.67 \% \\
\end{array}$ & $\begin{array}{c}46 \\
9.64 \% \\
\end{array}$ & $\begin{array}{c}60 \\
12.57 \% \\
\end{array}$ & $\begin{array}{c}26 \\
5.45 \% \\
\end{array}$ & $\begin{array}{c}63 \\
13.20 \% \\
\end{array}$ & $\begin{array}{c}48 \\
10.06 \% \\
\end{array}$ & $\begin{array}{c}54 \\
11.32 \% \\
\end{array}$ & $\begin{array}{c}477 \\
99.96 \% \\
\end{array}$ \\
\hline $\begin{array}{c}\text { Culex } \\
\text { quinquefasciatus }\end{array}$ & $9415.79 \%$ & $\begin{array}{c}85 \\
14.28 \% \\
\end{array}$ & $\begin{array}{c}51 \\
8.57 \% \\
\end{array}$ & $\begin{array}{c}43 \\
7.22 \% \\
\end{array}$ & $\begin{array}{c}66 \\
11.09 \% \\
\end{array}$ & $\begin{array}{c}71 \\
11.93 \% \\
\end{array}$ & $\begin{array}{c}74 \\
12.43 \% \\
\end{array}$ & $\begin{array}{c}53 \\
8.90 \% \\
\end{array}$ & $\begin{array}{c}58 \\
9.74 \% \\
\end{array}$ & $\begin{array}{c}595 \\
99.95 \% \\
\end{array}$ \\
\hline Culex vittatus & $\begin{array}{c}34 \\
21.38 \% \\
\end{array}$ & $\begin{array}{c}28 \\
17.61 \% \\
\end{array}$ & $\begin{array}{c}15 \\
9.43 \% \\
\end{array}$ & $\begin{array}{c}2 \\
1.25 \% \\
\end{array}$ & $\begin{array}{c}20 \\
12.57 \% \\
\end{array}$ & $\begin{array}{c}16 \\
10.06 \% \\
\end{array}$ & $\begin{array}{c}20 \\
12.57 \% \\
\end{array}$ & $\begin{array}{c}11 \\
6.91 \% \\
\end{array}$ & $\begin{array}{c}13 \\
8.17 \% \\
\end{array}$ & $\begin{array}{c}159 \\
99.95 \% \\
\end{array}$ \\
\hline $\begin{array}{c}\text { Eretmapodites } \\
\text { Species }\end{array}$ & $\begin{array}{c}26 \\
18.05 \% \\
\end{array}$ & $\begin{array}{c}19 \\
13.19 \% \\
\end{array}$ & $\begin{array}{c}26 \\
18.05 \% \\
\end{array}$ & $\begin{array}{c}16 \\
11.11 \% \\
\end{array}$ & $\begin{array}{c}2 \\
1.38 \% \\
\end{array}$ & $\begin{array}{c}20 \\
13.88 \% \\
\end{array}$ & $\begin{array}{c}13 \\
9.02 \% \\
\end{array}$ & $\begin{array}{c}12 \\
8.33 \% \\
\end{array}$ & $\begin{array}{c}10 \\
6.91 \% \\
\end{array}$ & $\begin{array}{c}144 \\
99.92 \% \\
\end{array}$ \\
\hline $\begin{array}{c}\text { Toxorhynchites } \\
\text { species }\end{array}$ & $\begin{array}{c}36 \\
32.72 \\
\end{array}$ & - & - & $\begin{array}{c}20 \\
18.18\end{array}$ & $\begin{array}{c}2 \\
8.18 \%\end{array}$ & $\begin{array}{c}18 \\
16.32 \% \\
\end{array}$ & $\begin{array}{c}15 \\
13.63 \% \\
\end{array}$ & $\begin{array}{c}8 \\
7.27 \% \\
\end{array}$ & $\begin{array}{c}4 \\
3.63 \% \\
\end{array}$ & $\begin{array}{c}110 \\
99.93 \%\end{array}$ \\
\hline TOTAL & $\begin{array}{c}432 \\
14.46 \%\end{array}$ & $\begin{array}{c}377 \\
12.62 \% \\
\end{array}$ & $\begin{array}{c}363 \\
9.80 \% \\
\end{array}$ & $\begin{array}{c}293 \\
10.54 \% \\
\end{array}$ & $\begin{array}{c}315 \\
11.88 \%\end{array}$ & $\begin{array}{c}355 \\
11.88 \% \\
\end{array}$ & $\begin{array}{c}355 \\
11.88 \% \\
\end{array}$ & $\begin{array}{c}244 \\
8.16 \%\end{array}$ & $\begin{array}{c}253 \\
8.47 \%\end{array}$ & $\begin{array}{c}2987 \\
99.96 \%\end{array}$ \\
\hline
\end{tabular}

\section{Discussion}

The results of the study clearly indicate a high occurrence of mosquitoes in the refuse dump sites in the study area. This could be attributed to the environmental conditions which favour the survival of the mosquito larvae in the various microhabitats. Similar results were obtained by previous workers [1, 2, 3, 4 and 9] in different parts of Nigeria. The environments of the immature stages and adult mosquitoes are interdependent [13] since the adult mosquitoes must have access to water for egg laying $[14,15]$.

The highest collection of mosquitoes was made during the month of April. This may be as a result of breeding of haemophilus insects at the onset and towards the end of the raining season when the organisms are not easily dislodged or swept by flood following heavy down pour in their breeding sites [16]. However there was presence of mosquitoes throughout the period under study due to the continuous rainfall which made water to be available in the breeding microhabitats in the refuse dumps. Disposal of refuse in Owerri urban has been a difficult task embarked by the Imo State Environmental Protection Agency (I.S.E.P.A). In most sites, refuse dumps are left for a long time before they were evacuated and these wastes are being removed; some parts of the dumps are left on the disposal sites. This paved way for microhabitats to retain water which gives ample opportunity for adult female mosquitoes to lay egg? The immature stages will also complete their life cycles in these microhabitats [17]. The water in the containers, tires and other breeding site are rain water collected after rainfall and were neither distorted, discarded nor dried up.

In site 1 which comprises of Relief Market and the surrounding environments, the refuse dump are not regularly evacuated and wastes are generated at a very high rate because of the nature of goods sold in this market. It is an urban market and as a result of this, the refuse are supposed to be disposed as soon as they are generated. This is also a high density area, and there are residential buildings many 
shops, schools and motor parks. All these factors give high rate of high rate of urbanization. Evacuation of the refuse dumps in this study site has not been on prompt basis.

In some dump sites (sites II, IV, VI, X, XII) the refuse dumps were observed to be about two month old which is long enough for mosquitoes to lay eggs that complete their life cycles in their microhabitats $[13,14]$.

Most of the drainage systems (gutter) in the site were blocked by wastes. This results to poor drainage which makes rain water in these drainage systems to per-collect thereby creating favorable microhabitats for mosquitoes that breed in polluted waters such Culex species [15]. Apart from Culex species that were breeding in the polluted waters, Anopheles mosquitoes were recorded in gutters and ground pools in most of the study sites. These ground pools were almost permanent and covered with vegetation. This is similar to studies by $[16,17]$ in Owerri, Abuja, Kaduna and Ogun States.

Human activities such as industrialization, commercialization and household activities in most of the study area gave rise to the rapid generation of wastes and their non-disposals, giving rise to the blockage of drainages that paved way for breeding and re-breeding of mosquitoes in the study area. Most of the study sites were water logged and the water body had remained undisturbed for a very long period. This also has resulted to the high abundance of mosquitoes. Site IV (Concord Hotel area) recorded the least in mosquito abundance. There is relatively high level of sanitation due to the higher social class of people living there. The occurrence of different species of mosquitoes shows that there may be a serious health risk of mosquitos' related diseases. The provision of conducive environment for the breeding of Anopheles gambiae complex, Culex species and Aedes species in the study area pre-disposes the population of diseases such as malaria, filariasis, encephalitis, dengue and yellow fever $[1,4]$ of which these mosquitoes are vectors [13]. Culex species complex present in the study area plays key role in the transmission of a range of pathogen including lymphatic filariasis, avian malaria, arbovirus and encephalitis [24]. The presence of these species of mosquitoes is made possible by their adaptation to human altered environment and these had led to their global distribution through disposals via humans [24]. Anopheles species identified in the breeding sites accounts for malaria endemicity in the study area. The Anopheles gambiae complex is also capable of transmitting filarial worms [23] and various arboviruses but malaria is unquestionably the most threatening [1]. Anopheles species complex was observed breeding in rain pools and ditches, used car tires, cans and gutters. This observation is similar to the results of $[8,9,26]$, in Awka, Enugu and Nasarawa. However swamps and cleaning standing waters were most preferred by these species.

Aedes aegypti, albopictus and vittatus of the subgenus Aedes stegomyia were observed in the study area. These are vectors of yellow fever as well as filariasis [27]. They are It is a mosquito of tropical and sub-tropical zones where they bites during the day time [28]. Majority of mosquitoes collected on the plants (tree holes) are members of the Aedes species.

Taeniorhynchus species mosquitoes also called elephant mosquitoes [25] were prevalent in the study area. It is among the few species of mosquitoes that are not of any medical importance. They do not suck blood but feed on nectar which helps in egg production. [25, 27]. However, the characteristics of water habitat whether it is running or standing, clean or polluted, shaded or sunlit, permanent or intermittent is the pre-dominant factor determining which species of mosquitoes breeds in it. Hence the presence of these identified mosquitoes species in the study area indicates the endemicity of mosquitoes-borne diseases.

There is need to develop prevention and control strategies for proper disposal of refuse by the public. The government should improve the standard of environmental sanitation, education on the proper disposed of refuse and construction of good drainage which facilitates system. This will help to minimize the blockage of gutters and stagnant water (pools) that encourage the blockage of water. Filling the potholes, low lying area and ditches will help to remove potential breeding sites. Refuse dumps should not be allowed to stay for a long time before taken to final disposal sites.

\section{REFERENCES}

[1] Anosike, J.C., Nwoke, B.E.B, Okere, A.N., Oku, E.E., Asor, J.E., Emmy-Egbe I.O., Adimike, D.A. (2007): Epidemiology of tree-hole breeding mosquitoes in the Tropical rain forest in Imo State, South East Nigeria. Ann Agric, Envi med 14, 31-38.

[2] Ugagu, mosquitoes of zoological Garden, Nekede, Imo state. Unpublished Thesis.

[3] Onyiudo, A.E., Azubuike, J., Amedi, E.S., Obiukwu, M.D., Ozumba, N.A., Ikpeze, O.O., (2011) A survey of public health disease vectors breeding in refuse dumps in onisha metropolis. New York Sue rice Journal 4(9).

[4] Onyiudo, A; Deezia, N; Obiukwu, M.D; Amadi, E., (2009): Ecology of man-biting mosquitoes in the developing sites of Nnamdi Azikiwe University Awka Anambra State Southern Nigeria. Internet Journal of Health (9)

[5] Mbanugo, J.I., and Okpalaononuju, C.N. (2013): Survellence of mosquitoes vectors in some habitat of Awka metropolis, Anambra State Nigeria Journal of Parasitology 24:184-190.

[6] Service, M.W. (1993): Mosquitoes (culicidae) In: medical insects and Arachnids. Lane, R.P. and R.W. cross key (Eds). Chapman and Hall, London p. 120-240.

[7] WHO (1975): Manual on practical Entomology in malaria, World Health Organization, Geneva. Prepared by the WHO division of malaria and other parasites diseases. Part 1-2 offset publication

[8] Michele, M., Cutiva, George, F.O., Mera (1938): University 
of Florida Entomology Laboratory Evans publishers.

[9] Gillet, J.D. (1972): Common African Mosquitoes and their medical impotence Willian Heinmann Medical books Limited; London $202 \mathrm{pp}$.

[10] Gillies, M.T, and Coetzee M. (1987). A supplement of the African Anopheline mosquitos south of Sahara.

[11] Service, M.W., (1990): Control of Urban Mosquitoes, Pesticide Outlook 1:17-20

[12] Gilles, M.T. and De Meillon 1968.

[13] Gilles, M.T, and Coetzee, M; (1968): A supplement to the Anophelinae of Africa South of the Sahara (Afro tropical Region).

[14] Ojiegbe, R.U., (2005): Characteristics and suitability of waste dumps in Owerri Nigeria. International Journal of National \& Applied Service 1:78-80.

[15] Service, M.W (1993): Mosquito (culicidae) in Medical insect and Aracnidas, Lane R.P. and Crosskey R.W., Ed Chapman and Hall, London p.120-140.

[16] Okoye, C., Nwachukwu, M.C.(2014): Human induce environmental vectors as mosquitoes breeding in Enugu urban, Nigeria. America Journal of Engineering Research 5(05) 57- 63.

[17] Farajollahi, A., Fonseca, D.M; Kilpatrick, M.A. (2011): Bird biting mosquitoes and human diseases. A review of the role of culex pipiens complex mosquitoes in epidemiology. Infection, Genetics Evolution 11(7): 1577-1585.

[18] Ahmed, A.B. (2011): Insect vectors of pathogens in selected indisposed refuse dumps in Kaduna town Northern Nigeria Service World Journal 6: 4.

[19] Okwa, O.O, Rasheed, A., Adeyemi, A., Omoyeri, M., Oni. (2006): Anopheles species of malaria patients reporting to hospitals in IJebu district of Ogun State Bulletin Science Association Nigeria 27: 23-31.

[20] Ozumba, N. A., Nwosu, E.O. (2003): Some implication of indispose in Enugu Urban South Eastern Nigeria. A preliminary solid waste epidemiology perspective study.

[21] Ogwueleke, T. (2009): Municipal Solid Waste Characteristics and management in Nigeria. Iran Journal of Environmental Science and Engineering 6(3) 173-180

[22] Savage, H.M., Kothera, L. (2012): The Culex pipiens complex in the Mississippi River Basin. Identification, Distribution and Blood Meal Hosts. Journal A.M Mosquitoes Control Association 28 (4) 93-99.

[23] Nasir, I. D., Muhammed, M.A., Emeribe, A.I, and Babayo, A. (2015): Prevalence of Malaria parasitemia among Residents Proximal to Environmental Waste Dump Sites in Abuja Nigeria. Journal of Medicine in the Tropics .17:91-96.

[24] Onwughara, I.N; Nnorom. I.C, Kanno, O.C. (2000): Issues of Road Side Disposal Habitat of Municipal Solid Waste, Environmental Impacts and Implementation of Sound Management practices in Developing Country Nigeria. International Journal of Environment Science and Development.

[25] Uwakwe, V. (2012): Solid waste management: A Case Study of Eneke, Port Harcourt, Rivers State World Press. 5: 19.

[26] Ayanda, O.I. (2009): Relative abundance of adult female anopheline mosquitoes in Ugah, Nasarawa State, Nigeria. Journal of vector-borne diseases 32:22-26.

[27] Onyiudo, A; Ozumba, N; Ezike, V, Nwosu, E, Chukwuekezie, O. and Amadi, E. (2009): Surveillance study of Aedes stegomyia Mosquitoes in the Ecological Locations of Enugu, south eastern Nigeria. Internet Journal of Infectious Diseases $8(1)$.

[28] Lee, V.H., Monath, R.P., Tomori, O., Fagbeni, A and Wilson, D.C. (1974): Arbovirus studies in Nupeko forests. A Possible Natural Forms of yellow Fever in Nigeria. Entomological Investigations. The Royal Tropical Medicine and Hygiene 68:39-43.

[29] Fagbani, J; Ahah, E.B; Fabiyi, A; Oconnor, E.H, (1975): Yellow fever outbreak in south western state of Nigeria. Virological and Serological Studies. Nigeria Medical Journal $6: 34-41$

[30] Tining Mosquitoes-dangers of Anopheles, Show fly system www.tiningmosquitoeanopheles.htm./

[31] Dengue and Aedes aegypti mosquitoes. www.cdc.gov/dengue/resources/30jan2012aegyptifactsheet.p df. 